

\section{A Quadrature Tau Method for Solving Fractional Integro-Differential Equations in the Caputo Sense}

\author{
A. Yousefi ${ }^{1, *}$, T. Mahdavi-Rad ${ }^{2}$, S.G. Shafiei ${ }^{1}$ \\ ${ }^{1}$ Department of Computer Science, Faculty of Mathematical Science and Computer, Kharazmi \\ University, Tehran, Iran \\ * (corresponding author) asadyosefi@gmail.com \\ ${ }^{2}$ Department of Mathematics, Arak branch, Islamic Azad University, Arak, Iran.
}

Article history:

Received November 2014

Accepted February 2015

Available online February 2015

\section{Abstract}

In this article, we develop a direct solution technique for solving fractional integro-differential equations (FIDEs) in the Caputo sense using a quadrature shifted Legendre Tau (Q-SLT) method. The spatial approximation is based on shifted Legendre polynomials. A new formula expressing explicitly any fractional-order derivatives of shifted Legendre polynomials of any degree in terms of shifted Legendre polynomials themselves is proved. Extension of the Tau method for FIDEs is treated using the shifted Legendre-Gauss-Lobatto quadrature. The method is illustrated by considering some examples whose exact solutions are available. The results obtained through this method are stable and comparable with the existing methods for a variety of problems with practical applications.

Keyword: Shifted Legendre Tau method, Fractional-order derivative, Caputo derivative, Fractional Integro-differential.

\section{Introduction}

Integro-differential equations are encountered as model in many fields of science and engineering such as population growth, one dimensional viscoelasticity and reactor dynamics [1-4]. Many mathematicians and applied researchers have tried to model real processes using the fractional calculus. Nigmatullin and Nelson described in terms of fractional kinetics in complex systems [5]. Jesus, Machado and Cunha analyzed the fractional order dynamics in botanical electrical impedances [6,7]. Petrovic, Spasic and Atanackovic developed a fractional-order mathematical model of a human root dentin [8]. In biology, it 
has been deduced that the membranes of cells of biological organism have fractional-order electrical conductance [10] and then are classified in groups of non-integer order models. Fractional derivatives embody essential features of cell rheological behavior and have enjoyed greatest success in the field of rheology [11]. Also, it has been shown that modeling the behavior of brainstem vestibule-oculumotor neurons by fractional ordinary differential equations (FODEs) has more advantages than classical integerorder modeling [9].

In this study, we consider a class of fractional integro-differential equations as follows

$$
L D(y)(x)=f(x)+\int_{0}^{t} k(x, s) D_{*}^{q} y(s) d s, \quad m-1<q \leq m, m \in \mathbb{N}, \quad t \in I=[0, t],
$$

with the initial condition

$$
y^{(i)}(0)=\beta_{i}, \quad i=0 \ldots n-1
$$

where $D_{*}^{q}$ is the fractional derivative operator given in the Caputo sense, $f(x)$ and $k(x, s)$ are the known functions that are supposed to be sufficiently smooth, $L$ is derivative operator in the form $L D(y)(x)=$ $\sum_{i=0}^{n} a_{i} D^{(i)} y(x)$ and $\beta_{i}$ for any $i$ is constant. M. F. Al-Jamal and E. A. Rawashdeh [12] present a numerical solution based on open Newton-Cotes formula. Existence and uniqueness of the solution of Eq. (1) have been shown in [13]. We want to consider the above FIDEs by using the spectral method.

Spectral methods provide a computational approach that has achieved substantial popularity over the last four decades. They have gained new popularity in automatic computations for a wide class of physical problems in fluid and heat flow. So, they have been applied successfully to numerical simulations of many problems in science and engineering (for more detail see [23]). Recently, Esmaeili and Shamsi [14] introduced a direct solution technique for obtaining the spectral solution of a special family of fractional initial value problems using a pseudo-spectral method. Neamaty and et al. [15] used Wavelet operational matrices for solving fractional differential equations. An extension of spectral methods for numerical solutions of some fractional differential equations is given in [16-19]. Moreover, Doha et al. [20] introduced a new efficient Chebyshev spectral algorithm for solving linear and nonlinear multi-term fractional orders differential equations. In fact, Doha et al. [21] used a quadrature Jacobi dual-PetrovGalerkin method for solving some ODEs. In the present paper, we construct the solution using the Q-SLT approach. This approach is based on the pseudo-spectral and Tau techniques. The fundamental goal of this paper is to propose a suitable way to approximate FIDEs in the Caputo sense using a quadrature shifted Legendre Tau approach. This approach extends the Tau method for FIDEs by approximating the weighted inner products in the Tau method by using the shifted Legendre-Gauss-Lobatto quadrature. This technique requires a formula for fractional-order derivatives of shifted Legendre polynomials of any degree in terms of shifted Legendre polynomials themselves which is also presented. Finally, examples are given to illustrate the efficiency and implementation of the method. Comparisons are made to confirm the reliability of the method.

The organization of this paper is as follows. In Section 2, some relevant properties of Legendre polynomials is explained. Also, we state and present a theory which gives explicitly a formula that expresses the fractional-order derivatives of the shifted Legendre polynomials of any degree in terms of the shifted Legendre polynomials themselves. In Section 3, we construct and develop an algorithm for solving FIDEs in the Caputo sense by using the Q-SLT method. In Section 4, the proposed method is applied to some examples. Also a conclusion is given in Section 5. 


\section{Fractional derivatives of shifted Legendre polynomials}

There are many definitions for fractional derivatives, and not necessarily equivalent to each other. The most commonly used definitions of fractional derivatives are the Riemann-Liouville and the Caputo definitions. By assuming $m \in \mathbb{N}$ is the smallest integer greater than or equal $q>0$, indeed $m$ is the integer satisfying the inequality $m-1<q \leq m$, and $\Gamma($.$) is the Euler's gamma function, we will have$ the following definitions.

Definition 2.1. The Riemann-Liouville operator of fractional differentiation of order $q$ is defined by

$$
D^{q} y(x)=\frac{d^{m}}{d t^{m}}\left(\frac{1}{\Gamma(m-q)} \int_{0}^{x}(x-z)^{m-q-1} y(z) d z\right), \quad x>0, \quad q \notin \mathbb{N}, q>0, m-1<q \leq m .
$$

Definition 2.2. The Caputo operator of fractional differentiation of order $q$ is defined by

$$
D_{*}^{q} y(x)=\frac{1}{\Gamma(m-q)} \int_{0}^{x}(x-z)^{m-q-1} y^{(m)}(z) d z, \quad x>0, \quad q>0, m-1<q \leq m .
$$

In this paper, we focus the Caputo operator of fractional differentiation, so let us write $D^{q}$ instead $D_{*}^{q}$.

Some important properties are (for further details, we refer to [20]):

$$
\begin{aligned}
& \text { - } D^{q} x^{v}=\left\{\begin{array}{cc}
\frac{\Gamma(v+1)}{\Gamma(v+1-q)} x^{v-q} & (v \in \mathbb{N} \text { and } v \geq m) \text { or }(v \notin \mathbb{N} \text { and } v>m-1), \\
0 & v \in\{0,1, \ldots, m-1\} .
\end{array}\right. \\
& \text { - } \quad D^{q}\left(\lambda y_{1}(x)+\mu y_{2}(x)\right)=\lambda D^{q} y_{1}(x)+\mu D^{q} y_{2}(x), \quad \lambda, \mu \in \mathbb{R} \text {. } \\
& \text { - } \quad D^{q} c=0, \quad(c \in \mathbb{R}) \text {. }
\end{aligned}
$$

Let $\mathrm{L}_{\mathrm{i}}(\mathrm{x})$ be the standard Legendre polynomial of degree $i$, then we have that

$$
L_{i}(-x)=(-1)^{i} L_{i}(x), \quad L_{i}(-1)=(-1)^{i}, \quad L_{i}(1)=1 .
$$

Let $w(x)=1$, then we define the weighted space $L_{w}^{2}(-1,1)$, equipped with the following inner product and norm

$$
(f, g)=\int_{-1}^{1} f(x) g(x) w(x) d x, \quad \quad\|f\|=(f, f)^{\frac{1}{2}}
$$

The set of Legendre polynomials forms a complete $L^{2}(-1,1)$ - orthogonal system, and

$$
\left\|L_{i}\right\|^{2}=\frac{2}{2 i+1}:=h_{i} .
$$

The shifted Legendre polynomial is defined as follow:

$$
L_{t, i}(x)=L_{i}\left(\frac{2 x}{t}-1\right), \quad t>0
$$

According to Eq. (3), it can be shown that

$$
L_{t, i}(0)=(-1)^{i}, \quad \frac{d^{q}}{d x^{q}} L_{t, i}(0)=\frac{(-1)^{i-q} \Gamma(i+q+1)}{t^{q} \Gamma(i-q+1) \Gamma(q+1)} .
$$


The analytic form of shifted Legendre polynomial of degree $i$ is given by

$$
L_{t, i}(x)=\sum_{k=0}^{i} \frac{(-1)^{i+k}(i+k) !}{(i-k) !(k !)^{2} t^{q}} x^{k} .
$$

We let $w_{t}(t)=w(t)=1$, then similarly the set of shifted Legendre polynomials forms a complete $L_{w_{t}}(-1,1)-$ orthogonal system. Due to Eq. (4) and Eq. (5), we will have

$$
(f, g)_{w_{t}}=\int_{-1}^{1} f(x) g(x) w_{L}(x) d x, \quad\|f\|_{w_{t}}=(f, f)_{w_{t}}{ }^{\frac{1}{2}} .
$$

and

$$
\left\|L_{t, i}\right\|_{w_{t}}^{2}=\frac{t}{2 i+1}=\frac{t}{2} h_{i}:=h_{t, i}
$$

A function $u(x)$, square integrable in $(0, t)$, may be expressed in term of shifted Legendre polynomial as

$$
u(x)=\sum_{k=0}^{\infty} a_{k} L_{t, k}(x)
$$

where the coefficients $a_{k}$ are given by

$$
a_{k}=\frac{1}{h_{L, k}} \int_{0}^{L} u(x) L_{t, k}(x) d x, \quad k=0,1,2, \ldots
$$

In practice, we can write

$$
u(x) \cong \sum_{k=0}^{N} a_{k} L_{t, k}(x)
$$

By using the Eq. (8), we have the following properties.

Lemma 2.1. let $L_{t, i}(t)$ be a shifted Legendre polynomial. Then

$$
D^{\alpha} L_{t, i}(x)=0, \quad i=0,1, \ldots,\lceil\alpha\rceil-1, \quad \alpha>
$$

0 .

Theorem 2.2. [22] The fractional derivative of order $\alpha$ in the Caputo sense for the Legendre polynomials is given by

$$
D^{\alpha} L_{t, i}(x)=\sum_{j=0}^{\infty} \mathrm{Z}_{\alpha}(i, j) L_{t, j}(x), \quad i=m, m+1, \ldots,
$$

where $Z_{\alpha}(i, j)=\sum_{k=m}^{i} \Omega_{i j k}$, and

$$
\Omega_{i j k}=(2 j+1) \sum_{r=0}^{j} \frac{(-1)^{i+k+j+r}(i+k) !(j+r) !}{t^{\alpha}(i-k) ! k !(j-r) !(r !)^{2} \Gamma(k-\alpha+1) \Gamma(k+r-\alpha+a)} .
$$




\section{A quadrature shifted Legendre Tau method}

In this section, we use the Q-SLT method to solve numerically Eq. (1) to gather Eq. (2) as follow:

We denote $\mathrm{x}_{\mathrm{N}, \mathrm{j}}\left(\mathrm{x}_{\mathrm{t}, \mathrm{N}, \mathrm{j}}\right)$ and $w_{N, j}\left(w_{t, N, j}\right), 0 \leq j \leq N$, the nodes and weights of the standard

(respectively shifted) Legendre-Gauss-Lobatto quadratures on the intervals $(-1,1)$ and $(0, t)$ respectively. Therefore one can easily show that

$$
\begin{array}{rlrl}
x_{t, N, j} & =\frac{t}{2}\left(x_{N, j}+1\right), & \\
w_{t, N, j}=\frac{t}{2} w_{N, j} . & 0 \leq j \leq N
\end{array}
$$

If $\mathrm{y}(x) \in \mathrm{P}_{2 \mathrm{~N}+1}(0, \mathrm{t})$, the set of all polynomials of degree at most $2 \mathrm{~N}+1$ in interval $(0, t)$, then

$$
\begin{aligned}
\int_{0}^{t} y(x) w_{t}(x) d x & =\frac{t}{2} \int_{-1}^{1} y\left(\frac{t}{2}(x+1)\right) w(x) d x \\
& =\frac{t}{2} \sum_{j=0}^{N} w_{N, j} y\left(\frac{t}{2}\left(x_{N, j}+1\right)\right) \\
& =\sum_{j=0}^{N} w_{t, N, j} y\left(x_{t, N, j}\right) .
\end{aligned}
$$

According to the Legendre-Gauss-Lobatto quadrature, $x_{N, j}$ are the zero of $\left(1-x^{2}\right) \partial_{x} L_{N}$, and

$$
w_{N, j}=\frac{2}{N(N+1)} \frac{1}{\left(L_{N}\left(x_{N, j}\right)\right)^{2}}, \quad 0 \leq j \leq N .
$$

We define the discrete inner product and norm as follows:

$$
(u, v)_{w_{t, N}}=\sum_{j=0}^{N} u\left(x_{t, N, j}\right) v\left(x_{t, N, j}\right) w_{t, N, j}, \quad\|u\|_{w_{t, N}}=\sqrt{(u, u)_{w_{t, N}}} .
$$

Obviously,

$$
(u, v)_{w_{t, N}}=(u, v)_{w_{t}}, \quad \forall u, v \in P_{2 N+1}
$$

Associating with this quadrature rule, we denote by $I_{N}^{S L}$ the shifted Legendre-Gauss-Lobatto interpolation,

$$
I_{N}^{S L} y\left(x_{t, N, j}\right)=y\left(x_{t, N, j}\right), \quad 0 \leq j \leq N .
$$

Theorem 3.1. [22] Given $y \in P_{N}$ and its values at a set of Legendre-Gauss-Lobatto points $\left\{x_{N, j}\right\}_{j=0}^{N}$, then we have

$$
\boldsymbol{y}^{(l)}=D^{l} \boldsymbol{y}
$$

where

$$
D=\left(d_{k j}\right)_{0 \leq j, k \leq N^{\prime}}, \quad \boldsymbol{y}^{(l)}=\left(y^{(l)}\left(x_{N, 0}\right), \ldots, y^{(l)}\left(x_{N, N}\right)\right)^{T}, \quad \boldsymbol{y}=\boldsymbol{y}^{(0)},
$$




$$
d_{k j}=\left\{\begin{array}{lr}
-\frac{N(N+1)}{4}, & k=j=0 \\
\frac{L_{N}\left(x_{N, k}\right)}{L_{N}\left(x_{N, j}\right)} \frac{1}{x_{N, k}-x_{N, j}}, & k \neq j, 0 \leq k, j \leq N \\
\frac{N(N+1)}{4}, & k=j=N \\
0 & 1 \leq k=j \leq N-1 .
\end{array}\right.
$$

Remark. In the case of $\alpha=n \in \mathbb{N}$, theorem 2.2 gives the same result as theorem 3.1.

The Q-SLT method for the FIDEs, (1)-(2), is to find $y_{N}(x) \in P_{N}$, such that

$$
\begin{aligned}
& \left(L D\left(y_{N}\right)(x), L_{t, k}(x)\right)_{w_{t}}=\left(f(x), L_{t, k}(x)\right)_{w_{t}}+ \\
& \left(\int_{0}^{t} k(x, s) D^{q} y(s) d s, L_{t, k}(x)\right)_{w_{t}} \\
& k=0,1, \ldots, N-n, \\
& y_{N}^{(i)}(0)=\beta_{i}, \quad i=0, \ldots, n-1 .
\end{aligned}
$$

By using Eq. (18), we employ a truncated series of shifted Legendre polynomials to approximate the unknown function, and the fractional-differential operator of this truncated series is expanded by shifted Legendre polynomials themselves (see, Theorem 2.2), and then the coefficients of this series are taken to be equal to the coefficients of the right-hand side expansion.

Let us denote

$$
\begin{aligned}
& y_{N}(x) \cong \sum_{j=0}^{N} c_{j} L_{t, j}(x), \quad \boldsymbol{C}=\left(c_{0}, c_{1}, \ldots, c_{N}\right)^{T} \\
& k(x, s) \cong \sum_{i=0}^{N} \sum_{j=0}^{N} k_{i j} L_{t, j}(s) L_{t, i}(x),
\end{aligned}
$$

such that

$$
k_{i j}=\left(\left(k(x, s), L_{t, j}(s)\right), L_{t, i}(x)\right)_{w_{t}} .
$$

then Eq. (22) can be written as

$$
\begin{gathered}
\sum_{j=0}^{N} c_{j}\left\{\left(L D\left(L_{t, j}(x), L_{t, k}(x)\right)_{w_{t, N}}-\left(\int_{0}^{t} k(x, s) D^{q} L_{t, j}(s) d s, L_{t, k}(x)\right)_{w_{t, N}}\right\}\right. \\
=\left(f(x), L_{t, k}(x)\right)_{w_{t, N}} .
\end{gathered}
$$

By using Theorem 2.2, we obtain 


$$
\begin{aligned}
& \sum_{j=0}^{m-1} c_{j}\left(L D\left(L_{t, j}(x), L_{t, k}(x)\right)_{w_{t, N}}\right. \\
& +\sum_{j=m}^{N} c_{j}\left\{\left(L D\left(L_{t, j}(x), L_{t, k}(x)\right)_{w_{t, N}}-\sum_{i=0}^{\infty} Z_{q}(j, i)\left(\int_{0}^{t} k(x, s) L_{t, i}(s) d s, L_{t, k}(x)\right)_{w_{t, N}}\right\}\right. \\
& =\left(f(x), L_{t, k}(x)\right)_{w_{t, N}} .
\end{aligned}
$$

Let us denote $\phi(x)=\left(L_{t, 0}(x), L_{t, 1}(x), \ldots, L_{t, N}(x)\right)^{T}$, then we can rewrite Eq. (26) as

$$
\begin{aligned}
& C^{T}\left(L D(\phi)(x), L_{t, k}(x)\right)_{w_{t}}-C^{T}\left(\left(\int_{0}^{t} D^{q} \phi(s) \phi^{T}(s) d s\right) \boldsymbol{K}^{T} \phi(x), L_{t, k}(x)\right)_{w_{t}} \\
& \cong \boldsymbol{F}^{T}\left(\phi(x), L_{t, k}(x)\right)_{w_{t}} .
\end{aligned}
$$

where

$$
\boldsymbol{K}=\left(k_{i j}\right)_{0 \leq i, j \leq N}, \quad \boldsymbol{F}=\left(f_{0}, f_{1}, \ldots, f_{N}\right)^{T}
$$

As in typical method, we generate $N-n+1$ linear equation by applying

$$
\left(R_{N}(x), L_{t, k}(x)\right)_{w_{t}}=0, \quad k=0,1, \ldots, N-n,
$$

where

$$
R_{N}(x)=\left(C^{T}\left(L D(.)-\left(\int_{0}^{t} D^{q} \phi(s) \phi^{T}(s) d s\right) \boldsymbol{K}^{T}\right)-\boldsymbol{F}\right) \phi(x) .
$$

Also, by substituting Eq. (24) and Eq. (21) into Eq. (23), we get

$$
y_{N}^{(k)}(0)=C^{T} D^{k} \phi(0)=\beta_{k}, \quad k=0,1, \ldots, n-1 .
$$

Eq. (29) and Eq. (31) generate $(N-n+1)$ and $n$ sets of linear equations, respectively. These linear equations can be solved for unknown coefficients of the vector $C$. Consequently, $y_{N}(x)$ given in Eq. (24) can be calculated, which gives the solution of the FIDEs problem in Eq. (1) and Eq. (2).

If we set $\alpha=q$ in Theorem 2.2, and we consider the only the first $(N+1)$-terms of shifted Legendre polynomials in Eq. (14), then we obtain

$$
\int_{0}^{t} D^{q} \phi(s) \phi^{T}(s) d s=\left(\left(D^{q} L_{t, j}, L_{t, k}\right)_{w_{t}}\right)_{0 \leq k, j \leq N},
$$

such that

$$
\begin{aligned}
\left(D^{q} L_{t, j}, L_{t, k}\right) & =\int_{0}^{t} D^{q} L_{t, j}(s) L_{t, k}(s) d s \cong \int_{0}^{t} \sum_{l=0}^{N} Z_{q}(j, l) L_{t, l}(s) L_{t, k}(s) d s \\
& =h_{t, k} Z_{q}(j, k), \quad k=0,1, \ldots, N-m, j=m, m+1, \ldots, N .
\end{aligned}
$$


where $Z_{q}(j, k)$ is given in Eq. (14).

\section{Illustrative examples}

To illustrate the effectiveness of the proposed algorithms, several illustrative examples are implemented by the method proposed in previous section. A comparison between the Q-SLT method and the method proposed by Al-Jamal et al. [12] is made. The results obtained by the proposed algorithms reveal that this algorithm is effective and convenient for approximating the IFDEs.

Example 1. Consider the IFDEs

$$
y^{\prime}(x)=14\left(1-\frac{t}{2.5 \Gamma(1.5)}\right)+\int_{0}^{1} x s D_{*}^{\frac{1}{2}} y(s) d s, \quad y(0)=0 .
$$

The exact solution is $y(x)=14 x$.

Now, we implement the Q-SLT technique with $N=1$, and then the approximate solution obtains as

$$
y_{1}(x)=c_{0}+c_{1} L_{1,1}(x)=c_{0}+c_{1}(2 x-1),
$$

By replacing the above relations in IFDEs, the following linear system yielded

$$
\left\{\begin{array}{c}
-10.840538332132564793+1.5486483331617949704 c_{1}=0 \\
c_{0}-c_{1}=0
\end{array}\right.
$$

By solving the recent system, we obtain $c_{0}=c_{1}=7$. Therefore

$$
y_{1}(x)=y(x)=14.000000000000000000 x \text {. }
$$

Example 2. Consider the equation

$$
y^{\prime}(x)=f(x)+\int_{0}^{1} k(x, s) D_{*}^{\frac{1}{4}} y(s) d s,
$$

with the initial condition

$$
y(0)=0,
$$

where $k(x, s)=x^{2} s^{2}, f(x)=8 x^{3}-\frac{3}{2} x^{\frac{1}{2}}-\left(\frac{48}{6.75 \Gamma(4.75)}-\frac{\Gamma(2.75)}{4.25 \Gamma(2.25)}\right) x^{2}$, and exact solution is given by $y(x)=2 x^{4}-x^{\frac{3}{2}}$

We let $N=6$ and obtain

$$
\begin{gathered}
y(x)=0.50 * 10^{-10}-0.1258741266 x-2.202797201 x^{2}+3.937048968 x^{3} \\
-3.286713287 x^{4}+3.776223777 x^{5}-1.076923077 x^{6} .
\end{gathered}
$$


A. Yousefi, T. Mahdavi-Rad, S.G. Shafiei/ J. Math. Computer Sci. 16 (2015) 97 - 107

Table 1: relative errors for Example 2 using the C.D.F-B.D.F and Q-SLT method at $t=0.5$

$\begin{array}{lcc}\mathrm{N} & \mathrm{R} \text {. Error at } t=0.5 \text { by C.D.F-B.D.F } & \text { R. Error at } \mathrm{t}=0.5 \text { by Q-SLT } \\ 6 & 0.85140834 & 0.01228875 \\ 12 & 0.03241737 & 0.01137419 \\ 18 & 0.12476347 & 0.01150399 \\ 24 & 0.00916074 & 0.00626470 \\ 30 & 0.04651995 & \\ & & \\ & & \\ & & \end{array}$

Rawashdeh and Al-Jamal in [12] use the Backward and Central-Difference Formulas (B.D.F-C.D.F) to approximate $D y(x)$ in Eq. (1) and approximate the solution $y(x)$ only at the mesh points. Table 1 and 2 compare the accuracy of C.D.F-B.D.F method and Q-SLT with relative errors.

Table 2: relative errors for Example 2 using the C.D.F-B.D.F and Q-SLT method at $t=1$

$\mathrm{N}$

6

12

18

24

30
R. Error at $t=1$ by C.D.F-B.D.F

0.51768770

0.15318017

0.07240337

0.04216677

0.02762368
R. Error at $\mathrm{t}=1$ by Q-SLT

0.02096505

0.02096351

0.02096348

0.02096125

0.00209604 


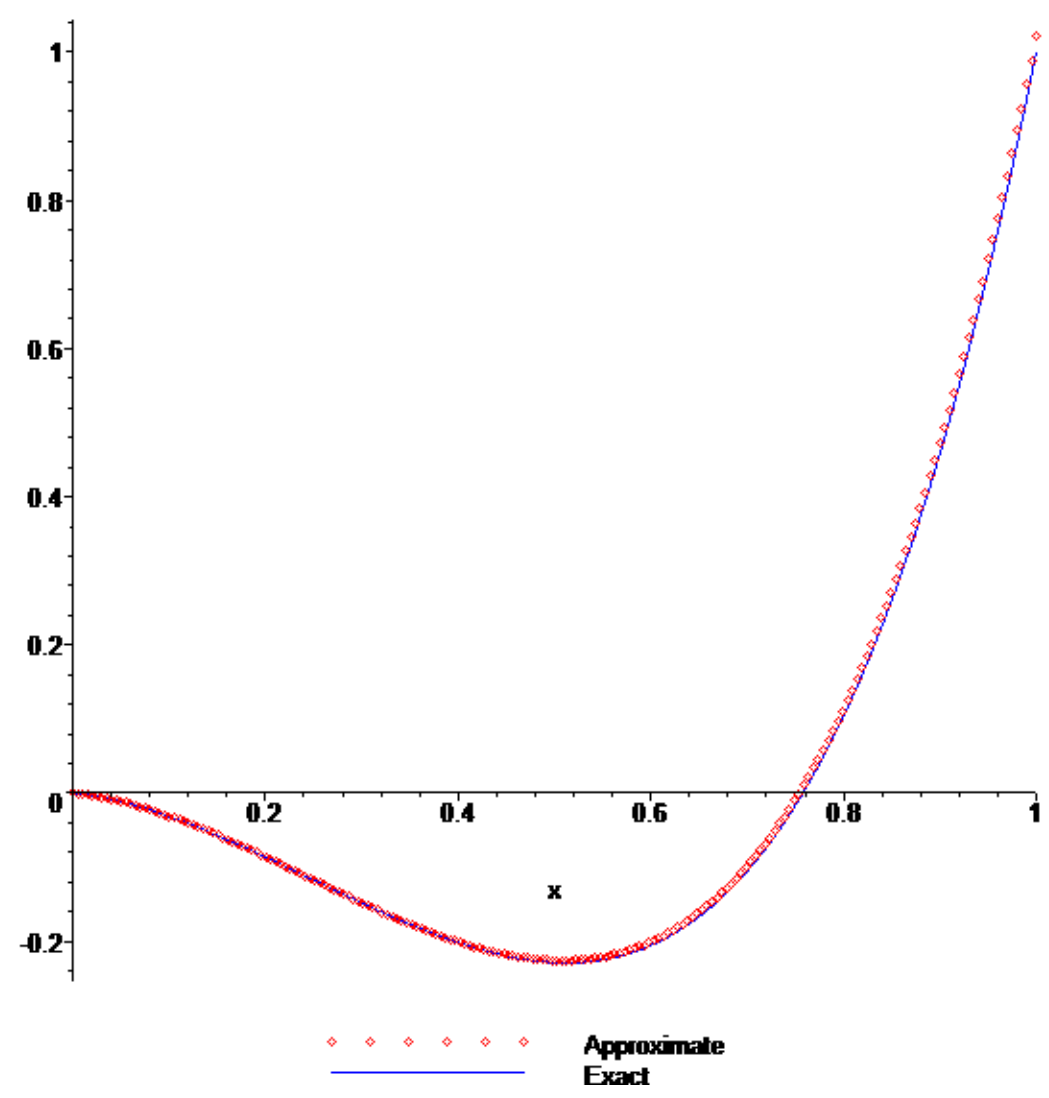

Figure 1: Comparison of the exact solution and our approximate solution for Example 2 with $N=6$

\section{Conclusion}

The presented method is based on computing the coefficients in the Legendre expansion of solution of a Fredholm integro-differential of fractional order. The Q-SLT method is useful both for finding the general solution and particular solution as demonstrated in examples. An interesting feature of this method is that when an integro-differential system has linearly independent polynomial solution of degree $\mathrm{N}$ or less than $\mathrm{N}$, our method can be used for finding the analytical solution. Besides, we see that there exists a solution which is closer to the exact solution if the truncation limit $\mathrm{N}$ is increased.

A considerable advantage of the method is that the Legendre coefficients the solution are found very easily by using the computer programs. Furthermore, the values of the solution at the collocation points are evaluated with the aid of the computer programs without any computational effort.

\section{Reference}

[1] M. Kot, "Elements of Mathematical Ecology", Cambridge University Press, (2001).

[2] D.-B. Pougaza, "The Lotka integral equation as a stable population model", Postgraduate Essay, African Institute for Mathematical Sciences (AIMS), (2007).

[3] I.D. Kopeikin, V.P. Shishkin, "Integral form of the general solution of equations of steady-state thermo elasticity", J. Appl. Math. Mech. (PMM U.S.S.R.). 48(1) (1984) 117-119.

[4] A.J. Lotka, "On an integral equation in population analysis", Ann. Math. Stat 10 (1939) 144161. 
[5] R.R. Nigmatullin, S.O. Nelson, "Recognition of the "fractional" kinetics in complex systems" Dielectric properties of fresh fruits and vegetables from 0.01 to $1.8 \mathrm{GHz}$, Signal Processing 86 (2006) 2744-2759.

[6] I.S. Jesus, J.A.T. Machado, J.B. Cunha, "Fractional electrical impedances in botanical elements", Journal of Vibration and Control 14 (2008) 1389-1402.

[7] I.S. Jesus, J.A.T. Machado, J.B. Cunha, "Fractional order electrical impedance of fruits and vegetables", in Proceedings of the 25th IASTED International Conference MODELLING, IDENTIFICATION, AND CONTROL, February 6-8, (2006), Lanzarote, Canary Islands, Spain.

[8] L.M. Petrovic, D.T. Spasic, T.M. Atanackovic, "On a mathematical model of a human root dentin", Dental Materials 21 (2005) 125-128.

[9] V.D. Djordjeviф, J. Jariф, B. Fabry, J.J. Fredberg, D. Stamenoviф, "Fractional derivatives embody essential features of cell rheological behavior", Annals of Biomedical Engineering 31 (2003) 692-699.

[10] K.S. Cole, "Electric conductance of biological systems", in Proc. Cold Spring Harbor Symp. Quant. Biol, Cold Spring Harbor, New York, (1993), pp. 107-116.

[11] T.J. Anastasio, "The fractional-order dynamics of bainstem vestibulo-oculomotor neurons", Biological Cybernetics 72 (1994) 69-79.

[12] M. F. Al-Jamal, E. A. Rawashdeh, "The approximate solution of fractional integro-differential equations", Int. J. Contemp. Math. Science, Vol.4, (2009), no.22, 1067-1078.

[13] W. G. El-Sayed and A. M.A. El-sayed, "On the fractional integral equations of mixed type integro-differential equations of fractional orders", Applied Mathematics and Computation, 154 (2004), 461-467.

[14] S. Esmaeili, M. Shamsi, "A pseudo-spectral scheme for the approximate solution of a family of fractional differential equations", Commun. Nonlinear Sci. Numer. Simul. 16 (2011) 36463654.

[15] A. Neamaty, B. Agheli, R. Darzi, "Solving fractional partial differential equation by using wavelet operational method", J. Math. Computer Sci. 7 (2013) 230 - 240.

[16] F. Ghoreishi, S. Yazdani, "An extension of the spectral Tau method for numerical solution of multi-order fractional differential equations with convergence analysis", Comput. Math. Appl. 61 (2011) 30-43.

[17] A. Pedas, E. Tamme, "On the convergence of spline collocation methods for solving fractional differential equations", J. Comput. Appl. Math. 235 (2011) 3502-3514.

[18] S.K. Vanani, A. Aminataei, "Tau approximate solution of fractional partial differential equations", Comput. Math. Appl. (2011) doi:10.1016/j.camwa.2011.03.013.

[19] M. Alipour, D. Rostamy, "Solving nonlinear fractional differential equations by Bernstein polynomials operational matrices", J. Math. Computer Sci. 3 (2012) 185-196.

[20] E.H. Doha, A.H. Bhrawy, S.S. Ezz-Eldien, "Efficient Chebyshev spectral methods for solving multi-term fractional orders differential equations", Appl. Math. Model. (2011) doi:10.1016/j.apm.2011.05.011.

[21] E.H. Doha, A.H. Bhrawy, R.M. Hafez, "A Jacobi dual-Petrov-Galerkin method for solving some odd-order ordinary differential equations", Abstr. Appl. Anal. (2011) doi:10.1155/2011/947230.

[22] A.H. Bhrawy, A.S. Alofi, S.S. Ezz-Eldien, "A quadrature Tau method for fractional differential equations with variable coefficients", App. Math. Letters 24 (2011) 2146-2152.

[23] J. Shen, T. Tang, Li-Lian Wang, "Spectral methods, Algorithms, Analysis and applications", Springer-Verlag Berlin Heidelberg (2011). 